\title{
Effects of Malaria Parasitaemia on Some Haematological Parameters of Pregnant Women of African Descent in Specialist Hospital Sokoto, North Western Nigeria
}

\author{
Osaro Erhabor ${ }^{1 *}$, Abdulrahaman Abdullahi ${ }^{1}$ and Erhabor Tosan ${ }^{2}$ \\ ${ }^{1}$ Department of Haematology, School of Medical Laboratory Science, Usmanu Danfodiyo University Sokoto, Nigeria \\ ${ }^{2}$ Medical Laboratory Science Council of Nigeria
}

Submission: March 24, 2019; Published: June 18, 2019

*Corresponding author: Osaro Erhabor, Department of Haematology, Usmanu Danfodiyo University Sokoto, Nigeria, School of Medical Laboratory Science, Usmanu Danfodiyo University Sokoto, Nigeria

\begin{abstract}
Despite the fact that malaria is a completely preventable disease, an estimated 3.4 billion people worldwide continue to be at risk of the disease globally. Malaria in pregnancy is a major contributor to adverse maternal and prenatal outcome. In hyper endemic areas like ours, it is a common cause of anemia in pregnancy and is aggravated by poor socioeconomic circumstance. This study evaluated the effect of Plasmodium falciparum parasitemia on some full blood count parameters among a total of 60 plasmodium parasitized pregnant women visiting the antenatal clinic in Sokoto Specialist Hospital in Sokoto, North Western Nigeria. Thirty malaria negative age-matched pregnant women were monitored as controls. A structured interviewer-administered questionnaire was used to obtain some socio-demographic characteristics of the participants. Blood samples was collected in Ethylene Diamine Tetra Acetic Acid (EDTA) tubes were examined for malaria parasite and some haematological parameters (haematocrit, haemoglobin, total white cell count, red blood cell count and platelet count) using the auto-haematology analyzer (Genesis, HA6000, China).

Data generated was analyzed using SPSS 25.0 statistical package. A p-value $\leq 0.05$ was considered significant in all statistical comparisons. There was a statistically significant decrease $(\mathrm{p}=0.000)$ in the packed cell volume (PCV), haemoglobin (HB), red blood cell count (RBC) and platelet counts of the parasitized subjects compared to the non-parasitized controls. Plasmodium falciparum was the predominant special responsible for all the cases of malaria among the subjects. Finding from this study has shown that malaria in pregnancy causes decrease in some of the haematological parameters. There is need for full blood count to be assayed in pregnancy and particularly among plasmodium parasitized women to facilitate the evidenced-based management of these women. Malaria parasite testing should be carried out routinely on pregnant women particularly those presenting to antenatal clinic with febrile illness. There is need for the Nigerian government to invest on evidenced-based best practices; education of pregnant women, provision of long-lasting insecticide-treated bed nets (LLINs), integration of malaria-prevention into antenatal care services and provision of prophylactic treatment with sulfadoxine-pyrimethamine (ITPp-SP) with two single doses taken after 16 weeks of amenorrhea, at least 4 weeks apart.
\end{abstract}

Keywords: Malaria Parasitaemia; Haematological Parameters; Pregnant Women; African; Specialist Hospital Sokoto; Nigeria

\section{Introduction}

An estimated 30 million women living in malaria endemic areas of Africa become pregnant each year. Pregnant women are particularly vulnerable to malaria because pregnancy reduces immunity to malaria and increases susceptibility to malaria infection. Malaria is associated with severe anaemia, acute pulmonary oedema, renal failure, puerperal sepsis, postpartum haemorrhage and increased risk of death. Malaria in pregnancy is associated with adverse pregnancy outcomes, such as spontaneous abortion, neonatal death, and low birth weight. Chronic anaemia, due to malaria may also affect a child's growth and intellectual development [1]. Malaria is the number one killer of all the parasitic diseases. It is estimated that at least 1 million people die of malaria particularly pregnant women and children under 5 years of age [2]. More than $80 \%$ of the deaths worldwide occur in sub-Saharan Africa. Malaria infection during pregnancy is a significant public health problem with substantial risk for the pregnant women, her foetus and the newborn child. Pregnant women and their unborn children are particularly vulnerable to malaria, which is a major cause of prenatal mortality, low birth weight and maternal anaemia. It accounts for $40 \%$ of public health expenditure, $30-50 \%$ of in-patient admissions and up to $50 \%$ of out-patient visits in areas with high malaria transmission [3].

Pregnancy appears to interfere with the immune processes in malaria, a disease which itself alters immune reactivity [4]. In highly endemic malarious areas where semi-immune adults usually have substantially acquired resistance to local strains of plas- 
modia, the prevalence of clinical malaria is higher, and its severity is greater in pregnant women than non-pregnant women [4]. Malaria transmission is increasing in Nigeria due to a number of reasons; deforestation, cultivation of wetlands, poor environmental sanitation, other man-made breeding sites such as construction works, brick pits or fishponds, among others, all of which create breeding sites for mosquito [5].

Haematological changes are some of the most common complications in malaria and they play a major role in malaria pathogenesis. These changes involve the major cell types such as RBCs, leucocytes and Platelets [6]. Malaria infected patients tended to have significantly lower platelets, WBCs, lymphocytes, Eosinophils, RBCs and Haemoglobin level, while Monocyte and Neutrophil counts were significantly higher in comparison to non-malaria infected patients [7].

Haematological changes during malaria infection, such as thrombocytopenia and leucocytosis or leucopenia are well recognized. Diagnostic value of these haematological alterations may be easily obtained and useful in people living in malaria endemic areas [8] reported that the most commonly changed parameters were platelet count, $\mathrm{Hb}, \mathrm{RBC}, \mathrm{MCV}, \mathrm{MCH}, \mathrm{MCHC}, \mathrm{WBC}$, neutrophil, and lymphocyte counts. Presence of thrombocytopenia in people from endemic areas may be useful as supportive diagnostic criteria for malaria in case with low level of parasite number.

Malaria is the most common cause of severe anaemia in endemic areas [9]. Anaemia in malaria is believed to occur due to haemolysis of parasitized and non-parasitized RBCs, peripheral removal/sequestration of RBCs, and ineffective erythropoiesis (due to high circulating tissue necrotic factor ( $\mathrm{TNF} \alpha$ ) [10]. In malaria endemic areas, the prevalence and severity of anaemia are usually determined by a number of interacting factors. These include, among others, the parasite species, level of parasitaemia, age of host, host genetic factors (coexisting RBC polymorphisms like haemoglobinopathies, G6PD), and non-malarial causes of anaemia (infection). As observed elsewhere [11], the mean red blood cell indices (Hb, MCV, MCH, MCHC, and RDW) of patients with acute uncomplicated malaria in this study were normal. This could probably have been because uncomplicated malaria is associated with milder biochemical changes, for example, a lower production of cytokines, less endothelial cell activation, milder changes in the coagulation profile, less sequestration, and less haemolysis as opposed to complicated/severe malaria.

Leukocytes play a vital role in the defense against malaria. Leukocyte changes in malaria are variable and depend on many factors such as acuteness of infection, parasitaemia, disease severity, state of the host immunity to malaria, and concurrent infections [9]. Commonly, majority of patients with acute uncomplicated $P$. falciparum malaria usually have their mean total leukocyte count (TLC) within the normal range [12]. However, in some cases, a mild leucopenia may occur, especially in non-immune adults or in cases of complicated malaria [13]. Finding from a retrospec- tive study done in a malaria endemic Indian province involving 334 patients with acute malaria caused by P. vivax, P. falciparum, or dual infection, there was a significant decrease in the mean TLC in the parasitaemia group [11]. One study showed patients with higher WBCs count compared with community controls [14]. Leukopenia is observed in majority of patients that had malaria caused by several different malaria parasite species $[15,16]$.

Lymphocyte count remains normal during an acute malaria infection [9] (Abdalla, 2004). Lymphopenia is sometimes profound but transient or temporary common finding in acute malaria in non-immune adults [17] as well as in children found in malaria endemic areas [6]. Monocytosis has been one of the most consistent observations reported from prior studies done on the haematological changes that characterize malaria [18]. Complicated and acute uncomplicated malaria is associated with normal neutrophil counts $[9,10]$. A few other studies that looked at eosinophils in malaria found low levels (eosinopenia) in majority of patients [19], although the significance of these findings was unknown. However, follow-up of these patients' days or weeks after treatment surprisingly revealed a marked elevation in the eosinophil count [19], a feature that the researchers attributed to the rebound eosinophilic response that resulted from of an enhanced $\mathrm{T}$ helper- 2 response that occurred during the malaria recovery period. The most common complication during malaria infection is thrombocytopenia [20]. Persons with platelet counts $<150,000 /$ $\mu \mathrm{L}$ were 12-15 times more likely to have malaria infection than persons with platelet counts $>150,000 / \mathrm{ml}$ [20]. Platelets and coagulation factors are vital components of the extraordinary complex environment that surrounds flowing or sequestered parasitized RBCs and the enclosing tubular vascular endothelium [9]. Because of that, a lot of research work has been dedicated to determining the effects of malaria on platelet homeostasis.

What is now apparent from those studies is the fact that thrombocytopenia is a major complication of malaria [21], the magnitude of which is dependent on the parasite species or disease severity. In light of the above, P. vivax malaria infection and severe malaria have been associated with a more heightened and severe thrombocytopenia than P. falciparum infection and uncomplicated malaria. In an attempt to compensate for the low absolute platelet count, the bone marrow increases the formation of megakaryocytes, which usually escape from the bone marrow as mega platelets during an acute malaria infection. Evidence to support this hypothesis comes from a study by Kreil et al. [22] that found a marked elevation in the level of thrombopoietin, a key platelet growth factor in patients with malaria. Because of an increase in the number of mega platelets, the mean platelet volume is increased during an acute malaria infection [6].

The pathogenesis of thrombocytopenia is thought to involve a constellation of processes, some of which include splenic pooling of platelets, antibody (IgG) mediated platelet destruction, adenosine diphosphate (ADP) release following the haemolysis of parasitized RBCs, dysmegakaryopoiesis, platelet aggregation and 
activation, parasite invasion of platelets, platelet phagocytosis, platelet adhesion to erythrocytes, and oxidative stress [23]. The relatively diverse causative pathophysiological mechanisms could probably explain why changes in platelet homeostasis are more prominent than in other blood cell lines. Nevertheless, thrombocytopenia in malaria is observed to improve with disease resolution, and a normal platelet count is usually reported within 7 days after the initiation of antimalarial treatment [24].

In Nigeria, Malaria is a major public health problem, where it accounts for more cases and deaths than any other country in the world [25]. Malaria is a risk for $97 \%$ of Nigeria's population, the remaining $3 \%$ of the population live in the malaria free highlands; there are an estimated 100 million malaria cases with over 300,000 deaths per year in Nigeria, it contributes to an estimated $11 \%$ of maternal mortality. It accounts for $60 \%$ of outpatient's visits and $30 \%$ of hospitalization among children under five years of age in Nigeria [25]. In Nigeria, malaria is endemic throughout with seasonal variation in different geographical zone of the country. More than $90 \%$ of the total population is at risk of malaria and at least $50 \%$ of the population suffers from at least one episode of malaria per year, beyond the impact on pregnant women and children it affects the population at large [25]. In Sokoto, there had been a number of researches on malaria [26-28] but there is probably no published information concerning the effect of malaria on the haematological of pregnant women in the research study area. A comprehensive study on the effect plasmodium infection among pregnant women in the locality is expected to provide baseline information, which will be useful in the effective formulation of adequate control measures, which could help move the locality towards achieving the Millennium Development Goals (MDGs). In addition, there is limited data on the result of prothrombin time, activated partial thromboplastin time and full blood count of pregnant women with malaria in Sokoto North Western Nigeria. The aim of this study is to determine the prothrombin time, activated partial thromboplastin time and full blood count parameters among pregnant women with malaria attending the antenatal clinic of Specialist Hospital Sokoto. The aim of this study is to determine the effect of malaria on some haematological and haemostatic parameters among pregnant women attending Specialist Hospital Sokoto in Sokoto Nigeria.

\section{Materials and Methods}

\section{Study Area}

This study was carried out in the Antenatal Clinic of Specialist Hospital, Sokoto, North-Western Nigeria. Specialist Hospital Sokoto is a tertiary institution located within the Sokoto metropolis. Sokoto is the capital city of Sokoto State of Nigeria. The State is located in the extreme Northwest of Nigeria, near to the confluence of the Sokoto River and the Rima River. The State is in the dry Sahel, surrounded by sandy savannah and isolated hills, with an annual average temperature of $28.3 \mathrm{oc}(82.9 \mathrm{oF})$. Sokoto is, on the whole, a very hot area. However, maximum daytime temperatures are for most of the year generally under 400c (104.00F) and the dryness makes the heat bearable. The warmest months are February to April when day time temperature can exceed 450c (113.0 $0 F$ ). The rainy season is from June to October during which shower are a daily occurrence. Sokoto city is a major commerce center in leather crafts and agricultural products. As at 2006, the state has a population of 3.6 million [29]. However, based on the population annual growth of $3 \%$, the calculated projected population for Sokoto State now stands at around 4.9 million.

\section{Study Population}

The study population for this study includes 60 malaria- infected pregnant women (subject) and 30 age- matched healthy non-parasitized pregnant women who were monitored as controls. Both subjects and controls ware recruited in the Antenatal Clinic of Specialist Hospital, Sokoto, Sokoto North-Western Nigeria.

\section{Study Subjects / Selection}

\section{Inclusion Criteria}

Women who meet the following inclusion criteria were recruited in the study;

a) Adult Pregnant women ( $\geq 18$ years) parasitized with plasmodium attending Antenatal Clinic Specialist Hospital, Sokoto, Nigeria.

b) Women in (a) who gave written informed consent in their clinic and agreed to be included in the study.

\section{Exclusion Criteria}

The following were excluded from participating as subjects in the study.

a) Non-adult pregnant women parasitized with plasmodium.

b) Healthy pregnant women that are not parasitized with plasmodium.

c) Plasmodium parasitized pregnant women who did not offer an informed consent to be included in the study.

\section{Study Design}

The research was a case-control study to assess the effect of malaria on some Haematological and Haemostatic Parameters of 60 Plasmodium parasitized pregnant women and 30 age and gender-matched healthy non-parasitized pregnant women were monitored as controls visiting the Antenatal Clinic Specialist Hospital, Sokoto. Blood sample were collected (from both subjects and controls) and tested for complete blood count, Prothrombin Time and Activated Partial Thromboplastin time.

\section{Sample Size Determination}

The sample size was determined using the standard formula for calculation of minimum sample size: 
$\mathrm{n} \quad=\quad$ minimum sample size

$\mathrm{z}=$ standard normal deviation and probability.

$\mathrm{p}=$ prevalence or proportion of value to be estimated from previous studies.

$\mathrm{q}=\quad$ Proportion of failure $(=1-\mathrm{P})$

$\mathrm{d}=$ precision, tolerance limit, the minimum is 0.05 .

Therefore, $\mathrm{n}=\mathrm{z}^{2} \mathrm{pq} / \mathrm{d}^{2}$

Where

$$
z=95 \%(1.96)
$$

$$
\begin{aligned}
& p=6.2 \%(0.062)[30] \\
& q=1-0.062(=0.938) \\
& d=5 \%(0.05)
\end{aligned}
$$

Therefore $n=(1.96)^{2}(0.062)(0.938) /(0.05)^{2} \quad n=88$

\section{Sample Collection}

Whole blood was collected via venipuncture, using BD vacutainer system into K3 EDTA anticoagulated and Tri-sodium citrate tube under strict aseptic techniques. The EDTA anticoagulated blood sample was used to analyze complete blood count while sample from the tri-sodium citrate tubes was centrifuged at $3000 \mathrm{rpm}$ for ten minutes on a bench-top centrifuge in order to get Platelet Poor Plasma (PPP). The PPP obtained was transferred into sterile tube and analyzed. These samples were tested in the Haematology Laboratory of Usmanu Danfodiyo University Teaching Hospital (UDUTH) Nigeria. The following laboratory investigations were carried out on K3EDTA and Citrated anticoagulated blood.

\section{Analytical Method}

\section{Diagnosis of Malaria}

Giemsa stain being one of the Romanowsky stain contains both acidic and basic components and thus can stain both the acidic and basic structures of the cell. When blood films are stained using Giemsa stain, the nucleus and cytoplasm of white blood cells takes on characteristic blue or pink colour. The full blood count was carried out using the Genesis HA6000 Auto Haematology Analyzer (Perlong Medical Equipment Company, China). The analyzer determines haematological parameters which include: Red Blood cell Count, Haemoglobin (HGB) Haematocrit (HCT), Total White cell Count (TWBC) and Platelet Count.

\section{Questionnaire}

A semi- structured interviewer-administered questionnaire was administered to all consenting participants in order to obtain information on their socio-demographic, nutritional and medical history. A sample of the questionnaire is shown in the appendix I.

\section{Informed Consent}

Written informed consent was obtained from all the study participants (subjects and controls). An example of the consent form used in this study is shown in the appendix II.

\section{Statistical Analysis}

Data obtained was entered into a statistical package (such as SPSS version 25) on a computer to define the nature of the distribution of data for each group. Statistical differences of data were analyzed using series of statistical analysis such as mean, standard deviation, Chi -square, student's t-test, ANOVA depending on the nature (categorical or continuous) and distribution of data (normal or non-normal). Pearson's correlation was used to determine the relationship between sets of data. Probability $(\mathrm{p} \leq 0.05)$ was used to determine the level of significant for all statistical analysis.

\section{Ethical Consideration}

Ethical approval for this study was obtained from the Ethical Committee of Specialist Hospital Sokoto (Reference number: SHS/

\begin{tabular}{|c|c|c|c|c|}
\hline Variables & Test(n=60) & Control(n=60) & $\mathrm{X} 2$ & p-value \\
\hline \multicolumn{5}{|c|}{ Age Group } \\
\hline $15-20$ & $8(13.3 \%)$ & $2(6.67 \%)$ & 2.5 & 0.645 \\
\hline $21-25$ & $22(36.7 \%)$ & $14(46.67)$ & & \\
\hline $26-30$ & $15(25 \%)$ & $8(26.67)$ & & \\
\hline $31-35$ & $11(18.3 \%)$ & $3(10 \%)$ & & \\
\hline $36-40$ & $4(6.7 \%)$ & $3(10 \%)$ & & \\
\hline \multicolumn{5}{|c|}{ Tribe } \\
\hline Hausa & $57(95 \%)$ & $25(83.3 \%)$ & 5.37 & 0.068 \\
\hline Fulani & $2(3.3 \%)$ & $5(16.7 \%)$ & & \\
\hline Yoruba & $1(1.7 \%)$ & $0(0 \%)$ & & \\
\hline \multicolumn{5}{|c|}{ Place of Residence } \\
\hline Urban & $58(96.7 \%)$ & $25(83.3 \%)$ & 4.957 & $0.026^{*}$ \\
\hline
\end{tabular}
SUB133/VOL.1 25 $5^{\text {th }}$ May 2018).

Results

Table 1: The Socio-demographic characteristics of test and controls. 
JOJ Nursing \& Health Care

\begin{tabular}{|c|c|c|c|c|}
\hline Rural & $2(3.3 \%)$ & $5(16.7 \%)$ & & \\
\hline \multicolumn{5}{|c|}{ Educational Status } \\
\hline Non-formal education & $29(48.3 \%)$ & $10(33.3 \%)$ & 9.04 & $0.029 *$ \\
\hline Primary & $8(13.3 \%)$ & $3(10 \%)$ & & \\
\hline Secondary & $18(30 \%)$ & $7(23.3 \%)$ & & \\
\hline Tertiary & $5(8.3 \%)$ & $10(33.3 \%)$ & & \\
\hline \multicolumn{5}{|c|}{ Occupation } \\
\hline Housewife & $52(86.7 \%)$ & $22(73.3 \%)$ & 2.432 & 0.296 \\
\hline Business & $6(10 \%)$ & $6(20 \%)$ & & \\
\hline Civil servant & $2(3.3 \%)$ & $2(6.7 \%)$ & & \\
\hline \multicolumn{5}{|c|}{ Use of Insecticide Treated Mosquito Net } \\
\hline Yes & $18(30 \%)$ & $28(93.3 \%)$ & 32.105 & $0.000^{*}$ \\
\hline No & $42(70 \%)$ & $2(6.7 \%)$ & & \\
\hline \multicolumn{5}{|c|}{ On Prophylactic Medication } \\
\hline Yes & $26(43.3 \%)$ & $0(0 \%)$ & 18.281 & $0.000^{*}$ \\
\hline No & $34(56.7 \%)$ & $30(100 \%)$ & & \\
\hline \multicolumn{5}{|c|}{ Gravidity } \\
\hline Prim gravidae & $14(23.3 \%)$ & $6(20 \%)$ & 2.057 & 0.357 \\
\hline Multigravida & 29 (48.3\%) & $19(63.3 \%)$ & & \\
\hline Grand multipara & $17(28.3 \%)$ & $5(16.7 \%)$ & & \\
\hline
\end{tabular}

The Full Blood Count of 60 malaria parasitized pregnant women (subjects) recruited from the Antenatal Unit of Specialist Hospital Sokoto and 30 apparently healthy pregnant women (controls) were analyzed. A structured interviewer administered questionnaire was used to obtain information of the subjects. Table 1 presents the socio-demographic characteristics of the Malaria parasitized pregnant subjects and controls. The prevalence of malaria was significantly higher in pregnant women resident in Urban area, those that do not use mosquito net, those that have no non-formal education and those that are not on prophylaxis medication $(\mathrm{p}<0.05)$. Age, tribe, occupation, and gravidity had no significant effect of the prevalence of malaria among pregnant women $(p>0.05)$.

Table 2 revealed that RBC, HGB, HCT and Platelet levels was significantly lower among malaria- parasitized pregnant subjects

tically significant difference in the WBC of malaria parasitized pregnant subjects compared to that of non-parasitized controls ( $p>0.05)$. All the full blood count parameters showed a significant positive correlation with the Haemostatic parameters $(p<0.05)$ Data are presented as mean \pm SEM for age and percentages for others. Figures in brackets are percentages of total Key: $t=t$-test, $\mathrm{x}^{2}=$ chi-square, SEM = Standard Error of Mean * = statistically significant. Data are presented as mean \pm SEM.

Key: RBC $=$ Red Blood Cells, HCT $=$ Haematocrit (Packed cell volume), $\mathrm{HGB}=$ Haemoglobin, $\mathrm{WBC}=$ white cell, $\mathrm{PLT}=$ Platelet, SEM $=$ Standard Error of Mean * = Statistically significant. Table 2 shows the difference in haematological parameters of the subjects and control, there is a statistically significant decrease in HCT, HGB and Platelet of the subject compared to the controls. However, the WBC count is within the normal reference range. as compared to controls $(\mathrm{p}<0.05)$. However, there was no statisTable 2: Mean comparison of some Haematological parameters for the test and controls.

\begin{tabular}{|c|c|c|c|c|}
\hline Parameter & Test $(\mathbf{n = 6 0})$ & Control $(\mathbf{n}=\mathbf{3 0})$ & t-test & p-value \\
\hline WBC $(\mathrm{x} 109 / \mathrm{l})$ & $7.4830 \pm 0.0944$ & $5.8967 \pm 0.1512$ & 9.279 & $0.000^{*}$ \\
\hline RBC $(\mathrm{x} 109 / \mathrm{l})$ & $3.6242 \pm 0.0348$ & $4.4613 \pm 0.1243$ & 8.343 & $0.000^{*}$ \\
\hline HGB (g/d) & $9.3783 \pm 0.0538$ & $10.8900 \pm 0.0946$ & -14.936 & $0.000^{*}$ \\
\hline HCT (\%) & $26.9283 \pm 0.1880$ & $29.4600 \pm .3051$ & -7.398 & $0.000^{*}$ \\
\hline PLT (x109/l) & $198.6333 \pm 1.6521$ & $273.7667 \pm 6.6752$ & -14.323 & $0.000^{*}$ \\
\hline
\end{tabular}

\section{Discussion}

Malaria is a major public health problem in sub-Sahara Africa including Nigeria, where it accounts for more cases of infection and death than other countries in the world and the extent of utilization of malaria preventive measures may impact on the burden of malaria in pregnancy [30,31]. The aim of this study was to investigate the effect of malaria on some Haematological and Haemostatic parameters in pregnant women attending antenatal clinic in Specialist Hospital Sokoto. A total of 90 pregnant women participated in this study. The subjects were aged 15-40 
years. Our finding is consistent with a previous report [4] in Sokoto which indicated that, young maternal age contributed to the seroprevalence of malaria parasitaemia among pregnant women.

Age has also been implicated as epidemiological studies have shown that malaria in pregnancy is more prevalent in younger than older age groups $[32,33]$. We observed that younger women in the age group 21-25 years constituted a significant number of the subjects (36.7\%) compared to older age group 36-40 (6.7\%). This finding is consistent with a previous report of Panti and Colleagues [34] who reported that majority of the asymptomatic malaria positive pregnant women (84\%) were aged between 20 and 34 years. Uneke and Colleagues [35] in Southern Nigeria also reported that individuals of age group 20-24 years had the highest prevalence of maternal malaria (52\%) while the least was recorded among those $>40$ years. This study has also found that use of insecticide- treated mosquito net has great influence in preventing malaria. Malaria prevalence was significantly higher among women who do not use insecticide treated mosquito nets $(p<0.05)$. This confirms the report of World Health Organization [36] on the use of insecticide treated mosquito nets as a means to reduce the lethal impact of malaria. In this study, the level of education was found to have influence on prevention of malaria in pregnancy. This finding is consistent with previous reports $[37,38]$ in Karachi, India and Maiduguri Nigeria respectively. This is suggestive that the level of education can play a role in preventing malaria infection.

This study has also found that use of insecticide- treated mosquito net has great influence in preventing malaria. Malaria prevalence was significantly higher among women who do not use insecticide treated mosquito nets $(\mathrm{p}<0.05)$. This confirms the report [36] that the use of insecticide treated mosquito nets as a means to reduce the lethal impact of malaria. In this study, the level of education was found to have influence on prevention of malaria in pregnancy. Majority of the malaria parasitized subjects had no formal education (48.3\%), this is followed by those who attained secondary level education $(30 \%)$ then followed by women educated to primary education (13\%) and tertiary level education $(8.3 \%)$ of the subjects. This finding is consistent with previous reports $[37,38]$ in Karachi, India and Maiduguri Nigeria respectively. This is suggestive that the level of education can play a role in preventing malaria infection. In this study, the predominant specie responsible for the cases of malaria was Plasmodium falciparum. Our finding is consistent with previous report [39-41]. Mapping the global distribution of malaria motivated by a need to define populations at risk for appropriate resource allocation, and to provide a robust framework for evaluating its global economic impact, has shown that malaria infection particularly caused by Plasmodium falciparum has been geographically restricted and remains entrenched in poor areas of the world particularly in sub Saharan Africa [42].

This study indicated that the packed cell volume (PCV), haemoglobin (HGB) and red blood cell count (RBC) were significantly lower among the plasmodium parasitized subjects compared to controls $(\mathrm{p}<0.05)$. This finding is consistent with previous reports [30] which indicated that infected patients tended to have significantly lower haemoglobin and red blood cell count. The lower HCT, HGB and RBC may reflect anaemia which is mainly due to mechanical destruction of parasitized red blood cells, nutritional status of the infected pregnant women as well as splenic clearance of parasitized and defected red cells. Our finding agrees with previous report carried out in Sokoto State, Nigeria by Garba et al. [43], which indicated that PCV is lower in malaria infected pregnant women than the non-infected.

We observed that the mean platelet count was significantly lower $(\mathrm{p}<0.05)$ in malaria parasitized pregnant women compared to controls. The most common complication during malaria infection is thrombocytopenia [20]. Persons with platelet counts $<150,000 / \mu \mathrm{L}$ were $12-15$ times more likely to have malaria infection than persons with platelet counts $>150,000 / \mathrm{ml}$ [20]. Thrombocytopenia is a major complication of malaria [21], the magnitude of which is dependent on the parasite species or disease severity. In an attempt to compensate for the low absolute platelet count, the bone marrow increases the formation of megakaryocytes, which usually escape from the bone marrow as mega platelets during an acute malaria infection. Evidence to support this hypothesis comes from a study by Kreil et al. [22] that found a marked elevation in the level of thrombopoietin, a key platelet growth factor in patients with malaria. Because of an increase in the number of mega platelets, the mean platelet volume is increased during an acute malaria infection [6]. The pathogenesis of thrombocytopenia is thought to involve a constellation of processes, some of which include splenic pooling of platelets, antibody (IgG) mediated platelet destruction, adenosine diphosphate (ADP) release following the haemolysis of parasitized RBCs, dysmegakaryopoiesis, platelet aggregation and activation, parasite invasion of platelets, platelet phagocytosis, platelet adhesion to erythrocytes, and oxidative stress [23]. Thrombocytopenia in malaria is observed to improve with disease resolution, and a normal platelet count is usually reported within 7 days after the initiation of antimalarial treatment [24].

We observed that the mean total white cell count was significantly higher among plasmodium falciparum parasitized subjects compared to non-parasitized controls. The mean values of WBC count in both infected and non-infected women were within normal reference range, This may be due to the fact that the white blood cell is responsible for body defence during pregnancy as a result of building immunity for the foetus, immune response against malaria infection and immuno modulation in the presence of microbial activity, which is in contrast with the work of Osonuga and Colleagues [44] who reported that WBC was elevated in malaria-parasitized pregnant women and that this may be due to the fact that white blood cell are responsible for the body defence during pregnancy. Leukocytes play a vital role in the defense against malaria. Leukocyte changes in malaria are variable and depend on many factors such as acuteness of infection, par- 
asitaemia, disease severity, state of the host immunity to malaria, and concurrent infections [9]. Commonly, majority of patients with acute uncomplicated $P$. falciparum malaria usually have their mean total leukocyte count (TLC) within the normal range [12]. However, in some cases, a mild leucopenia may occur, especially in non-immune adults or in cases of complicated malaria [13]. Finding from a retrospective study done in a malaria endemic Indian province involving 334 patients with acute malaria caused by $\mathrm{P}$. vivax, P. falciparum, or dual infection, there was a significant decrease in the mean TLC in the parasitaemia group [11]. One study showed patients with higher WBCs count compared with community controls [14]. Leukopenia is observed in majority of patients that had malaria caused by several different malaria parasite species [15-16].

\section{Conclusion}

Findings of this study has shown that there is a significant decrease in HCT, HGB, RBC and Plateletcount of malaria-parasitized pregnant women subjects. We recommend that pregnant women presenting to antenatal clinic with febrile illness should be tested for malaria. There is need for the Nigerian government to invest in evidenced-based best practices; implementation of malaria prevention and control strategies, especially among high-risk pregnant women; increased enrolment of females in the area in school so as to improve access to formal education, provision of long-lasting insecticide-treated bed nets (LLINs), integration of malaria-prevention into antenatal care services and provision of prophylactic treatment with sulfadoxine-pyrimethamine (ITPpSP) with two single doses taken after 16 weeks of amenorrhea, at least 4 weeks.

\section{References}

1. World Health Organization Severe Malaria (2006) WHO. Tropical Medicine and International Health 1 (6): 7-131.

2. WHO (2005) World Health Organization, Geneva. WHO World Malaria Report, Geneva, Switzerland.

3. Bruce MC, Macheso A, Galinski MR \& Barnwell JW (2006) Characterization and application of multiple genetic markers for Plasmodium malariae. Parasitology 134(5): 637-650.

4. Saidu AY, Sadiya H, Dikwa MA, Abubakar MM, Fana SA, et al. (2015) Detection of Plasmodium Species among Pregnant Women attending Antenatal Care. IOSR Journal of Dental and Medical Science14(11): 61-66.

5. WHO (2005b) World Health Organization, Geneva. WHO World Malaria Report.

6. Maina RN, Walsh D, Gaddy C, Hongo C, Waitumbi J, et al. (2010) Impact of Plasmodium falciparum infection on haematological parameters in children living in Western Kenya. Malaria Journal 9: 43-54.

7. Bakhubaira S (2013) Haematological parameters in severe complicated Plasmodium falciparum malaria among adults in Aden. Turkey Journal of Haematology 30: 394-399.

8. Manas K, Bhukdee P, Nuoil P, Chaowanee C, Suwit D (2014) Effect of malarial infection on haematological parameters in population near Thailand-Myanmar border. Malaria Journal 13: 218.

9. Abdullah SPG (2004) Malaria: A Haematological perspective. Imperial College Press London, United Kingdom: 21-27.
10. Akhtar S, Gumashta R, Mahore S \& Maimoon S (2012) Haematological changes in malaria: a comparative study. Journal of Pharmacy and Biological Sciences 2(4): 15-19.

11. Chandra S, Chandra H (2013) Role of haematological parameters as an indicator of acute malarial infection in Uttarakhand State of India. Mediterranean Journal of Hematology and Infectious Diseases 5(1): e2013009.

12. Haroon H, Fazel PA, Naeem M, Mobin A, Naqvi AH, et al. (2013) Hide and seek: haematological aspects of malaria-a developing country perspective. Journal of Infection in Developing Countries 7(3): 273279.

13. Taylor WRJ, Widjaja H, Basri H (2008) Changes in the total leukocyte and platelet counts in Papuan and non-Papuan adults from northeast Papua infected with acute Plasmodium vivax or uncomplicated Plasmodium falciparum malaria. Malaria Journal 7: 259.

14. Ladhani S, Lowe B, Cole AO, Kowuondo K \& Newton CR (2002) Changes in white blood cells and platelets in children with falciparum malaria: relationship to disease outcome. British Journal of Haematology 119: 839-847.

15. Muwonge H, Kikomeko S, Sembajjwe LF, Seguya A, Namugwanya C (2013) How Reliable Are Hematological Parameters in Predicting Uncomplicated Plasmodium falciparum Malaria in an Endemic Region? ISRN Trop Med 2013: 1-9.

16. Koltas IS, Demirhindi H, Hazar S \& Ozcan K (2007) Supportive presumptive diagnosis of Plasmodium vivax malaria: thrombocytopenia and red cell distribution width. Saudi Medical Journal 28(4): 535-539.

17. Richards MW, Behrens RH \& Doherty JF (1998) Short report: Hematologic changes in acute, imported Plasmodium falciparum malaria. American Journal of Tropical Medicine and Hygiene 59(6): 859.

18. Abdalla SH (1988) Peripheral blood and bone marrow leucocytes in Gambian children with malaria: numerical changes and evaluation of phagocytosis. Annals of Tropical Paediatrics 8(4): 250-258.

19. Davis TME, Ho M, Supanaranond W, Looareesuwan S, et al. (1991) Changes in the peripheral blood eosinophil count in falciparum malaria. Acta Tropical 48(3): 243-246.

20. Erhart LM, Yingyuen K, Chuanak N, Buathong N, Laoboonchai A, et al (2004) Hematologic and clinical indices of malaria in a semi-immune population of western Thailand. American Journal of Tropical Medicine and Hygiene 70(1): 8-14.

21. Khan SJ, Abbass Y \& Marwat MA (2012) Thrombocytopenia as an indicator of malaria in adult population. Malaria Research and Treatment.

22. Bhasin TS, Sharma S, Manjari M (2013) Changes in megakaryocytes in cases of thrombocytopenia: bone marrow aspiration and biopsy analysis. J Clin Diagn Res 7(3): 473-479.

23. Lacerda MVG, Mourão MPG, Coelho HC, Santos JB (2011) Thrombocytopenia in malaria: who cares? Memorias do Instituto Oswaldo Cruz 106(1): 52-63.

24. Moulin F, Lesage F, Legros AH, Maroga C, Maussavou A, et al. (2003) Thrombocytopenia and Plasmodium falciparum malaria in children with different exposures. Archives of Disease in Childhood 88(6): 540-541.

25. World Health Organization (2012) Indoor residual spraying: use of indoor residual spraying for scaling up global malaria control and elimination 3: 49.

26. Umar RA, Hassan SW (2001) The relationship between the levels of parasitaemia and anaemia in children with malaria. Sahel Medical Journal 5: 58-62.

27. Jiya NM (2001) Cerebral malaria: Report in children 6 months to 12 
years at Usmanu Danfodiyo University Teaching Hospital, Sokoto. Dissertation submitted to FWACP.

28. Elueze EI, Osisanya JO \& Edafiogho IO (1990) Sensitivity to chloroquine in vivo and in vitro of plasmodium falciparum in Sokoto, Nigeria Transactions of the Royal Society of Medicine and Hygiene 84(1): 45.

29. NPC/FGN (2006) Nigerian Population Commission, Federal Republic of Nigeria. Special FGN Gazette No. 23 on the 2006 Population Census.

30. Udomah FP, Isaac I Z, Lukman I, Nwobodo D, Erhabor O, et al. (2015) Plasmodium parasitemia among pregnant women attending antenata clinic in Sokoto, North western Nigeria. Journal of Nursing Sciences 1 (1): 9-14.

31. Tango 00, Orimadegun AE \& Akinyinka 00 (2012) Utilization of malaria preventive measures during pregnancy and birth outcomes in Ibadan, Nigeria. BMC Pregnancy and Childbirth 11: 1-7.

32. Nwagha UI, Ugwu VO, Nwagha TU and Anyaehie USB (2009) Asymptomatic Plasmodium parasitaemia in pregnant Nigerian women: almost a decade after Roll Back Malaria. Translation of Royal Society of Tropical Medicine and Hygiene 103: 16-20.

33. Bouyou-Akotet MK, Lonete-Collard DE, Mabika-Manfoumbi M, Kendjo E, Matsiegui P B et al. (2003) Prevalence of Plasmodium falciparum infection in pregnant women in Gabon. Malaria Journal 2: 18.

34. Panti A A, Omokanye L O, Ekele B A, Jiya N M A, Isah A Y, et al. (2010) The prevalence of asymptomatic malaria parasitemia at delivery in Usmanu Danfodiyo University Teaching Hospital Sokoto. Global Research Journal of Medical Sciences 2(4): 048-053.

35. Uneke C J, Sunday-Adeoyeb I, Iyarec F E, Ugwujad E I \& Duhlinskae D D (2007) Impact of maternal Plasmodium falciparum malaria and haematological parameters on pregnancy and its outcome in southern Nigeria. Journal of Vector Borne Diseases 44: 285-290.

This work is licensed under Creative Commons Attribution 4.0 License DOI: 10.19080/JOJNHC.2019.10.555795
36. World Health Organization (2011). World malaria report fact sheet.

37. Ali S S, Karim N, Billoo AG \& Haider S S (2005) Association of literate mothers with malnutrition under three years of age in rural areas of district Malir, Karachi. Journal of Pakistan Medical Association 55: 550553.

38. Hamidu J L, Salami HA, Ekanem AU \& Hamman L (2003) Prevalence of protein energy malnutrition in Maiduguri, Nigeria. African Journal of Biomedical Research 6: 123-125.

39. Erhabor 0, Azuonwu O, Frank-Peterside N (2012) Malaria parasitaemia among long distance truck drivers in the Niger delta of Nigeria. Afr Health Sci 12(2): 98-103.

40. Jeremiah ZA, Jeremiah TA, Emelike FO (2010) Frequencies of some human genetic markers and their association with Plasmodium falciparum malaria in the Niger Delta, Nigeria. J Vector Borne Dis 47(1): $11-16$

41. Erhabor O, Jeremiah ZA, Adias TC, Hart ML (2010) Thrombocytopenia in plasmodium parasitized pregnant women in the Niger Delta of Nigeria. Pathology and Laboratory Medicine International 2: 1-5.

42. Snow RW, Guerra CA, Noor AM, Myint HY, Hay SI (2005) The global distribution of clinical episodes of Plasmodium falciparum malaria. Nature 434(7030): 214-217.

43. Garba N, Danladi SB \& Muhammed A (2015) Determination of some Haematological parameters in malaria infected subjects in Usmanu Danfodio University Teaching Hospital (UDUTH) Sokoto, Nigeria. Bayero Journal of Pure and Applied Sciences 8(1): 80-83.

44. Osonuga O, Onadeka AA, \& Osonuga A (2011) Haematological profile of pregnant women in Southwest of Nigeria. Asian Pacific Journal of Tropical Disease 1(3): 232-234.

\section{Your next submission with Juniper Publishers} will reach you the below assets

- Quality Editorial service

- Swift Peer Review

- Reprints availability

- E-prints Service

- Manuscript Podcast for convenient understanding

- Global attainment for your research

- Manuscript accessibility in different formats

( Pdf, E-pub, Full Text, Audio)

- Unceasing customer service

Track the below URL for one-step submission https://juniperpublishers.com/online-submission.php 